\title{
Laser Fluorescence
}

National Cancer Institute

\section{Source}

National Cancer Institute. Laser Fluorescence. NCI Thesaurus. Code C60724.

A method used to aid in the detection of tooth decay by measuring fluorescence using a laser. 